\title{
ESTUDIO DE LA CONTAMINACIÓN POR METALES PESADOS EN LAS COSTAS DE TACNA - PERÚ
}

Responsable: Mgr. Walter Ibárcena Fernández

\section{RESUMEN}

La costa peruana se han visto sometidas desde hace mucho tiempo, especialmente en las últimas 4 décadas, a una fuerte presión por contaminación humana e industrial, lo que ha afectado seriamente a la integridad del ecosistema. En este trabajo se hace una recopilación de información en las costas de Tacna, del nivel que tienen en la actualidad algunos metales pesados ( $\mathrm{Cd}, \mathrm{Cu}, \mathrm{Zn}, \mathrm{Pb}, \mathrm{Mg}$ ) en el agua marina, sedimentos y recursos marinos.

Por lo tanto, es necesario analizar el estado toxicológico en que se encuentran estos ecosistemas, obteniendo datos actuales del nivel de contaminación por metales pesados a través del análisis de estas zonas marinas, más aún cuando muchos recursos de la zona son utilizados para la alimentación humana. En el Perú, y especialmente en la zona sur, las descargas domésticas y mineras ocasionan mayor contaminación en las aguas receptoras, las primeras por el volumen de sus vertimientos, y las segundas por el vertimiento de sustancias tóxicas.

\section{ABSTRACT}

Peruvian coast they have seen submitted for a lot of time, specially in the last 4 decades, to a strong pressure for human and industrial pollution, which it has affected seriously to the integrity of the ecosystem. In this work a summary of information is done on the coasts of Tacna, of the level that have at present some heavy metals (CD, Cu, $\mathrm{Zn}$, $\mathrm{Pb}, \mathrm{Mg}$ ) in the marine water, sediments and marine resources.

Therefore it is necessary to analyze the toxicological condition in which they find these ecosystems, obtaining current information of the level of pollution by metals weighed across the analysis of these marine zones, even more when many resources of the zone are used for the human nourishment. In Peru, and specially in the south zone, the domestic and mining unloads, they cause major pollution in the waters sea-coasts, the first ones for the volume of his unloads, and the second ones, for the unloads of toxic substances.

\section{INTRODUCCION}

Para el Perú, con sus 3080 kilómetros de litoral, la zona costera reviste una singular importancia, ya que además de los recursos energéticos y minerales que posee, representa también una fuente significativa de de alimentos. Sin embargo, en la actualidad las bahías, estuarios y lagunas costeras del país encaran serios problemas de contaminación, los cuales producen daños considerables a los organismos que los habitan.

Los ecosistemas costeros y estuarios son sumamente frágiles, y a lo largo del litoral del país han ido sufriendo transformaciones muy relevantes, ocasionadas por las represas de los ríos, vertimiento de desechos municipales e industriales de las poblaciones colindantes, los cuales contienen diversos contaminantes entre los que destacan algunos metales pesados como Hg, $\mathrm{Cd}, \mathrm{Pb}, \mathrm{Cr}$, entre otros.

En consecuencia, la estimación de las concentraciones naturales y de los aportes antropogénicos de los metales pesados permite evaluar el nivel en que se encuentra afectada una zona. Para tal propósito es indispensable analizar, tanto la columna de agua como los sedimentos y los organismos, especialmente aquellos de hábitos bentónicos $y$ filtradores, los cuales han sido extensamente empleados como indicadores de contaminación (Villanueva et al., 1992).

Los metales pesados generalmente forman parte de los elementos constituyentes del agua de mar y se originan por los procesos de vulcanización e hidrotermalismo y de la erosión de las rocas, que durante el ciclo biogeoquímico natural se interrelacionan con el ambiente a través de los desechos y material sedimentario proveniente de las actividades industriales, en muchos casos esta movilización antropogénica excede a la movilización natural (Arias de Díaz et al., 2001).

\section{MATERIALES Y METTODOS}

\section{Muestreo:}

- Ubicación de la Zona de Muestreo: Situada en tres zonas específicas del litoral de Tacna, como se puede apreciar en la Figura 1, se fijaron 3 zonas de muestreo, Z1: Una ubicada al sur del foco contaminado, Z2: en el foco contaminado, y Z3: al norte del foco contaminado.

Figura. 1: Ubicación de las Zonas de Muestreo 


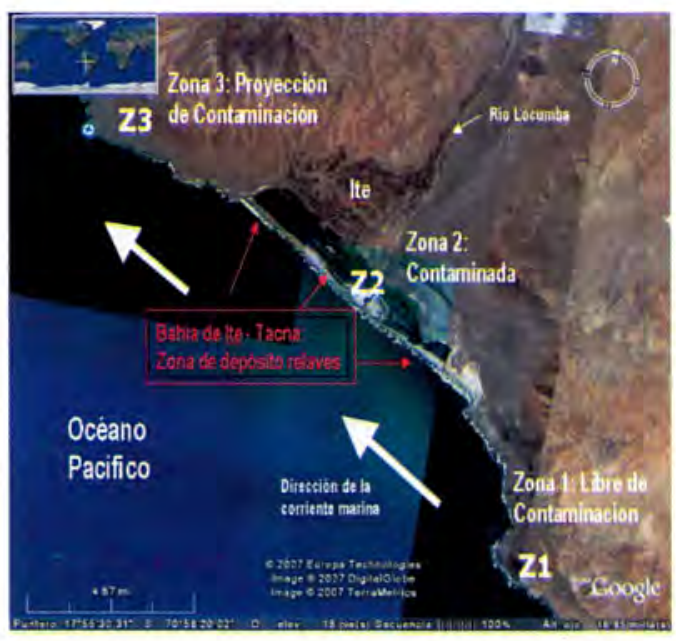

La zona de muestreo 01, al Sur del foco contaminado (Z1), que estuvo compuesta de 09 estaciones de muestreo, estuvo ubicada en la zona de Punta Mesa, donde se fijaron 03 perfiles de trabajo para las 9 estaciones de muestreo.

La zona de muestreo 02 ubicada en Playa Inglesa, (Z2) no se pudo ejecutar por la imposibilidad de ingreso a la zona, por restricciones impuestas por la Empresa Southern Perú.

La zona de muestreo 03 al norte del foco contaminado (Z3), que estuvo compuesta de 09 estaciones de muestreo, estuvo ubicada en la zona de Santa Rosa, donde se fijaron 03 perfiles de trabajo para las 9 estaciones de muestreo.

- Toma de Muestras y Traslado: Las muestras de agua de mar y sedimentos marinos fueron congeladas y luego enviadas a la Sede Central, del Instituto del Mar del Perú (IMARPE), donde se realizó el proceso de análisis. Finalmente, las lecturas analiticas se realizaron en el espectrofotómetro de absorción atómica modelo 6701F-Shimadzu con sistema automatizado en horno de grafito y flama.

\section{- Equipos y Material de Vidrio: Equipos y Material:}

- Espectrofotómetro de absorción atómica modelo 6701F-Shimadzu

- Balanza analítica

- Cocina eléctrica

- Destilador de agua

- Refrigerador

- Erlenmeyer, fiolas, pipetas, embudos, balones, vasos de precipitado, etc.

- Salinometro.

- Botella Niskin.

\section{RESULTADOS}

1. Zona de estudio: Santa Rosa (Zona 1):

El monitoreo se realizó en el área de estudio que se encuentra localizada, en el banco natural de Punta Mesa, al sur de la Bahía de Ite (entre los $18^{\circ} 03^{\prime} 8.4^{\prime \prime}$ y $18^{\circ} 04^{\prime}$ $17.4^{\prime \prime}$ LS $70^{\circ} 48^{\prime} 10,2^{\prime \prime}$ y $70^{\circ} 46^{\prime} 34.2^{\prime \prime}$ LW) en la provincia de Jorge Basadre, Tacna. (Figura $\mathrm{N}^{\circ} 01$ ).

Resultado de los análisis de metales pesados en agua de mar se muestran en la Tabla $N^{\circ} 01$, los cuales resultan del promedio del resultado de los análisis de metales pesados en agua de mar en la zona de Punta Mesa, ubicada al sur de la Bahía de Ite (foco contaminado), en tres estaciones de muestreo, en cada estación fue realizado el análisis por triplicado, haciendo un total de 09 análisis, 03 por cada estación. Para la determinación de metales pesados se aplicó la metodología de espectrometría absorción atómica. También se presenta la varianza, desviación estándar, intervalos de confianza de los cuatro metales analizados.

Tabla 01: Parámetros estadísticos aplicados a los resultados de los análisis de metales pesados en agua de mar, en Punta Mesa.

\begin{tabular}{|c|c|c|c|c|c|}
\hline $\begin{array}{l}\text { Parámetro } \\
\text { Estadistico }\end{array}$ & Simbolo & $\begin{array}{l}\text { Cadmio } \\
\text { (ug/L) }\end{array}$ & $\begin{array}{l}\text { Plomo } \\
\text { (ug/L) }\end{array}$ & $\begin{array}{l}\text { Cobre } \\
\text { (ugll.) }\end{array}$ & $\begin{array}{l}\text { Hierro } \\
\text { (ug/L) }\end{array}$ \\
\hline Media & $x$ & 0,530 & 0,720 & 10,66063519 & 0,360 \\
\hline \begin{tabular}{|l} 
Varianza \\
\end{tabular} & $S^{2}$ & 0 & 0,008533333 & 1,9939 & 0 \\
\hline Desviac. Estandar & $\mathrm{s}$ & 0 & 0.092376043 & 1.41205524 & 0 \\
\hline Desviac. Media & $\begin{array}{l}?(x,-= \\
x)\end{array}$ & 0 & 0,011 & 14,06659043 & 0 \\
\hline Num. Muestra & $\mathrm{N}$ & 3 & 3 & 3 & 3 \\
\hline Int. de Confianza & & $\begin{array}{l}0.530 \\
0.530\end{array}$ & $\begin{array}{l}0.615041 . \\
0.824108\end{array}$ & $\begin{array}{l}9,062744 \\
12.268526 \\
\end{array}$ & $\begin{array}{l}0,36 \cdot \\
0,36\end{array}$ \\
\hline
\end{tabular}

Fuente: IMARPE Laboratorio Regional Ilo.

Tabla 02: Parámetros estadísticos aplicados a los resultados de los análisis de metales pesados en sedimentos de mar, en Punta Mesa.

\begin{tabular}{|c|c|c|c|c|c|c|c|}
\hline $\begin{array}{l}\text { Parametro } \\
\text { Estadistic } \\
0\end{array}$ & $\begin{array}{c}\text { Simb } \\
\text { olo }\end{array}$ & $\begin{array}{l}\text { Cadmilo* } \\
\text { (ug/g) }\end{array}$ & mo* & \begin{tabular}{|c|} 
Mangane \\
so* \\
(ug/m)
\end{tabular} & & $\begin{array}{l}\text { Zinct } \\
(u g / g)\end{array}$ & $\begin{array}{l}\text { Fierro* } \\
\text { (ug/g) }\end{array}$ \\
\hline Media & $X$ & & 10 & & & & \\
\hline & $s^{2}$ & & & 4042,2470 & $\begin{array}{r}35,530133 \\
33\end{array}$ & & \\
\hline & & $\begin{array}{r}0.045825 \\
757\end{array}$ & $\begin{array}{r}0.16643 \\
317\end{array}$ & \begin{tabular}{|r|}
63,578668 \\
07
\end{tabular} & $\begin{array}{r}5.9607158 \\
41\end{array}$ & $\begin{array}{r}5,3794702 \\
34\end{array}$ & $\begin{array}{r}0,97277 \\
9523\end{array}$ \\
\hline $\begin{array}{l}\text { Des } \\
\text { Med }\end{array}$ & & & & & & & \\
\hline & $\mathrm{N}$ & & & & & & \\
\hline & & $\begin{array}{l}0,1081 . \\
0,2118\end{array}$ & $\begin{array}{l}1,2516- \\
1,6283\end{array}$ & \begin{tabular}{|c|}
$55.5972-$ \\
19948 \\
\end{tabular} & $\begin{array}{l}33,268- \\
46,758\end{array}$ & $\begin{array}{l}8,731- \\
18,467\end{array}$ & $\begin{array}{l}0,6991- \\
2,9008\end{array}$ \\
\hline
\end{tabular}

*Referido a muestra seca

Fuente: IMARPE Laboratório Regional llo.

El promedio del resultado de los análisis de metales pesados en sedimentos de mar en la zona de Punta Mesa se presenta en la Tabla $N^{\circ} 02$, ubicada al sur de la Bahía de Ite (foco contaminado), en tres estaciones de 
muestreo, en cada estación fue realizado el análisis por triplicado, haciendo un total de 09 análisis, 03 por cada estación. Para la determinación de metales pesados se aplicó la metodología de espectrometria absorción atómica. También se presenta la varianza, desviación estándar, intervalos de confianza de los metales analizados.

2. Zona de Estudio: Playa Ite (Zona 2):Los muestreos en dicha zona no se ejecutaron por las restricciones en el acceso impuestas por la empresa Southern Perú a toda persona particular que desee ingresar al área del litoral de la zona de estudio.

3. Zona de estudio: Santa Rosa (Zona 3):Se realizó el monitoreo en el área de estudio que se encuentra localizada, en el banco natural de Santa Rosa, al norte de la Bahía de Ite (se localizó entre los $17^{\circ} 52^{\prime} 42.1^{\prime \prime}$ y $17^{\circ} 53^{\prime} 0.2^{\prime \prime}$ LS $71^{\circ} 03^{\prime} 23,4^{\prime \prime}$ y $71^{\circ} 01^{\prime} 58^{\prime \prime}$ LW) en la provincia de Jorge Basadre, Tacna. (Figura 01).

Resultado de los análisis de metales pesados en agua de mar, en las estaciones de muestreo de Santa Rosa, Tabla 03, ubicadas al norte de la Bahía de Ite (foco de contaminación), obtenido del promedio por triplicado de cada estación de muestreo, haciendo un total de 09 análisis, 03 de cada estación de muestreo. Para la determinación de metales pesados se aplicó la metodología de espectrometría absorción atómica. También se presenta la varianza, desviación estándar, intervalos de confianza de los cuatro metales analizados.

Tabla 03: Parámetros estadísticos aplicados a los resultados de los análisis de metales pesados en agua de mar, en Santa Rosa.

\begin{tabular}{|c|c|c|c|c|c|}
\hline $\begin{array}{l}\text { Parámetro } \\
\text { Estadistico }\end{array}$ & Simbolo & $\begin{array}{l}\text { Cadmio } \\
\text { (ug/L) }\end{array}$ & $\begin{array}{l}\text { Plomb } \\
\text { (ug/L) }\end{array}$ & $\begin{array}{l}\text { Cobre } \\
\text { (ug/L) }\end{array}$ & $\begin{array}{l}\text { Hierro } \\
\text { (ugl) }\end{array}$ \\
\hline Meda & $X$ & 0,530 & 0,711 & 13,42837335 & 1,005 \\
\hline Vanianza & $\mathrm{s}^{2}$ & $\overline{0}$ & 0,00563333 & 4.806 & 76,8955333 \\
\hline Desviac.Estandar & $\bar{S}$ & $\overline{0}$ & 0,075055555 & 2.192357939 & 8,769015528 \\
\hline Desviac. Mecia & $?\left(X_{1}-X\right)$ & 0 & 0 & 0 & \\
\hline Num. Muestra & $N$ & 3 & 3 & 3 & 3 \\
\hline Int. de Corfiarza & & $0,53-0,53$ & $0.6258-0.7867$ & 10,9474159092 & $-8,31815-11,52738$ \\
\hline
\end{tabular}

Fuente: IMARPE Laboratorio Regional llo

Promedio del resultado de los análisis de metales pesados en sedimentos de mar en la zona de Santa Rosa, Tabla 04, ubicada al norte de la Bahía de Ite (foco contaminado), en tres estaciones de muestreo, en cada estación fue realizado el análisis por triplicado, haciendo un total de 09 análisis, 03 por cada estación. Para la determinación de metales pesados se aplico la metodología de espectrometria absorción atómica.
También se presenta la varianza, desviación estándar, intervalos de confianza de los cuatro metales analizados.

Tabla 04: Parámetros estadísticos aplicados a los resultados de los análisis de metales pesados en sedimentos de mar, en Santa Rosa.

\begin{tabular}{|c|c|c|c|c|c|c|c|}
\hline $\begin{array}{l}\text { Parametro } \\
\text { Estadistico }\end{array}$ & Simbolo & \begin{tabular}{|c} 
Cadmio* \\
(uglg)
\end{tabular} & $\begin{array}{l}\text { Plomot } \\
\text { (ug/g) }\end{array}$ & \begin{tabular}{|c}
$\begin{array}{c}\text { Manganeso* } \\
(\mathrm{ug} / \mathrm{m})\end{array}$ \\
\end{tabular} & $\begin{array}{l}\text { Cobre } \\
(\omega \mathrm{w} / \mathrm{g})\end{array}$ & $\begin{array}{l}\text { Zine } \\
\text { (ug/g) }\end{array}$ & $\begin{array}{l}\text { Ferro" } \\
\text { (vig/g) }\end{array}$ \\
\hline Meda & $X$ & 0,328 & 1,200 & 71,5488121 & 157.568 & \begin{tabular}{|l|}
9.945083329 \\
\end{tabular} & 1.410 \\
\hline Varianze & $s^{2}$ & $\begin{array}{r}0.0050535 \\
333 \\
\end{array}$ & 0,022533333 & 174,7262333 & 2499,164233 & 24.6544 & $\begin{array}{l}0,7212353 \\
33\end{array}$ \\
\hline $\begin{array}{l}\begin{array}{l}\text { Deswisc Estat } \\
\text { ndar }\end{array} \\
\end{array}$ & s & $\begin{array}{r}0.036149 \\
546 \\
\end{array}$ & 0,150443788 & 13.21848075 & 49,99164163 & 4.965319728 & $\begin{array}{r}0.3481857 \\
74 \\
\end{array}$ \\
\hline $\begin{array}{l}\text { Deswac } \\
\text { Necia }\end{array}$ & $2\left(x_{1}-x\right)$ & 0.026 & 0.019 & 2.42356 & 14,875 & 2.745 & 0.081 \\
\hline \begin{tabular}{|l|} 
Num. \\
Muestra
\end{tabular} & $\mathrm{N}$ & 3 & 3 & 3 & & 3. & i \\
\hline $\begin{array}{l}\text { Int de } \\
\text { Confarza }\end{array}$ & & $\begin{array}{l}0,2169 \\
0.4390 \\
\end{array}$ & $\begin{array}{l}1,03006- \\
1.3705 \\
\end{array}$ & $\begin{array}{l}56,5006- \\
86,5069\end{array}$ & $\begin{array}{l}100,997 . \\
214,1391 \\
\end{array}$ & $4,326-15.563$ & $\begin{array}{l}1.015 \\
1,803 \\
\end{array}$ \\
\hline
\end{tabular}

${ }^{\star}$ Referido a muestra seca

Fuente: IMARPE Laboratorio Regional Ilo.

\section{DISCUSION DE RESULTADOS}

Sobre la base de los resultados obtenidos de los análisis de metales pesados en agua de mar, en las estaciones de muestreo de Punta Mesa (Zona de muestreo 1), ubicadas al sur de la Bahía de Ite (foco de contaminación), obtenido del promedio por triplicado en cada estación, de un total de 09 análisis, 03 de cada estación de muestreo, se han obtenido niveles bastante bajos de metales pesados, siendo el más elevado el de Cobre con 12,34 $\mu \mathrm{g} / \mathrm{l}$.

El promedio de resultados obtenidos en agua de mar en la Zona de Punta Mesa para el cadmio es $0,53 \mu \mathrm{g} / \mathrm{l}$, según la FDA un ingreso crónico de cadmio puede llevar a la disfunción del hígado, la que debe estar entre 5 y $6 \mu \mathrm{g} / \mathrm{g}$ (Calvario. 2003). El nivel de contaminante para agua potable por debajo de $5 \mu \mathrm{g} / \mathrm{l}$, no se esperan riesgos para la salud (Juárez 2006). Por lo tanto, en la zona de muestreo los niveles de $\mathrm{Cd}$ alcanzado no significan riesgo para la salud. De esta manera se puede indicar que las concentraciones máximas de cadmio son muy inferiores al estándar EPA $(5,0 \mathrm{ppb})$ y superiores al promedio de las concentraciones máximas naturales $(0,05 \mathrm{ppb})$ (Chuecas $\mathrm{M}$. 1989)

El resultado obtenido para el plomo en el agua de mar fue de $0,72 \mu \mathrm{g} / \mathrm{l}$. La Ley general de aguas D.L: 17752 , en su Item V, Aguas de zonas de pesca de mariscos y bivalvos, establece límites de $10 \mu \mathrm{g} / \mathrm{l}$. Pero la FDA ha establecido que niveles de plomo en moluscos desde 1,5 a $6,3 \mu \mathrm{g} / \mathrm{g}$ afectan al sistema nervioso (Calvario 2003). En áreas costeras del Golfo de México, se obtuvieron valores más altos de plomo en agua en los años de 1982 a 1984 , con concentraciones de 29,4 y $11,6 \mathrm{mg} / \mathrm{l}$ respectivamente (Villanueva et al, 1992). 
En la Zona 1, los niveles de Cobre obtenido en el agua de mar fueron de $10,66 \mu \mathrm{g} / \mathrm{l}$, en las Costa de Galicia, España, se encontraron valores de 0,14 a $0,88 \mu \mathrm{g} / \mathrm{l}$ de $\mathrm{Cu}$ en agua de mar (Pérez López et al 2003). La Ley general de aguas D.L: 17752 , y D.S. N ${ }^{\circ}$ 261-69-AP, en su Item $\mathrm{V}$, Aguas de zonas de pesca de mariscos $\mathrm{y}$ bivalvos, establece limites de $10 \mu \mathrm{g} / \mathrm{l}$, para el cobre.

Hierro encontrado en el agua de mar fue de $0,36 \mu \mathrm{g} / \mathrm{l}$, nivel relativamente bajo para la zona de estudio. En general, las concentraciones de metales disueltos y en suspensión decrece al aumentar la salinidad. Este hecho es observable sobre todo a valores de salinidad menores a $18 \%$ (Cabrera F. et al, 1978). En nuestro caso, en la zona de toma de muestras se tiene una salinidad de $34,745 \%$. La legislación venezolana establece concentraciones limites de $179 \mu \mathrm{mol} / \mathrm{l}$ para aguas de descargas y $5,36 \mu \mathrm{mol} / \mathrm{l}$ para aguas destinadas a consumo humano (Márquez $\mathrm{A}$. et al, 2000). A diferencia de muchos contaminantes, los metales pesados son constituyentes normales del ambiente marino y, aun cuando en trazas, son generalmente detectados. Este hecho puede ser un significativo problema para diferenciar las concentraciones naturales detectadas en el ambiente de aquellas producidas por procesos de contaminación (Chuecas, M, 1989).

Según los resultados de los análisis de metales pesados en sedimentos marinos, en las estaciones de muestreo de Punta Mesa, ubicadas al sur de la Bahía de Ite (foco de contaminación), obtenido del promedio por triplicado de cada estación de muestreo, de un total de 09 análisis, 03 de cada estación de muestreo, se obtuvo el nivel más alto en manganeso con $164,50 \mu \mathrm{g} / \mathrm{g}$, y en segundo lugar el cobre con $46,84 \mu \mathrm{g} / \mathrm{g}$.

El promedio de cadmio encontrado en los sedimentos marinos fue de $0,16 \mu \mathrm{g} / \mathrm{g}$, valores por debajo de los límites permisibles, esto puede deberse a la baja cantidad de materia orgánica en las zonas de muestreo, existiendo más arenilla que material de caracteristicas orgánicas. Valores altos de cadmio están asociados con las características del sedimento y al contenido de materia orgánica, el cual forma compuestos orgánicos con el cadmio, asi como también de los desechos industriales (INGGO 1980, Rosas et al., 1983, Álvarez et al., 1986., Botello y Paez-Ozuna 1986).

El plomo en sedimentos en la zona de estudio fue de 1,44 $\mu \mathrm{g} / \mathrm{g}$. En el Golfo de México se encontraron valores que oscilan desde valores no detectables hasta concentraciones de $158,68 \mu \mathrm{g} / \mathrm{g}$, (Villanueva et al., 1992), lo que significa que en la Zona 1, la que suponemos libre de contaminación la cantidad de plomo encontrada es baja. Esto puede ser debido a que Punta Mesa presenta mayor influencia de las corrientes que circulan de sur a norte, las cuales transportan un buen volumen de metales pesados, en forma disuelta y particulada fuera de la zona. Este nivel de $\mathrm{Pb}$ promedio encontrado está en el rango reportado por $\mathrm{WHO}$, que es de $110 \mathrm{mg} / \mathrm{kg}$ (Amat, et al. 2002).

Niveles de manganeso de $127,54 \mu \mathrm{g} / \mathrm{g}$ se encontraron en sedimentos marinos. No existen criterios claros que permitan establecer la contaminación por metales pesados de un sedimento, porque la composición de los sedimentos puede ser muy variada, dependiendo de la roca madre, de la granulometria, del contenido de materia orgánica, etc. (Cabrera F. et al., 1987). Concentraciones de manganeso en el estuarios de Barbate (España) presentan 294,1 $\mu \mathrm{g} / \mathrm{g}$., estuario de Guadalquivir (España) 468,2 $\mu \mathrm{g} / \mathrm{g}$. (Cabrera F. et al., 1988).

El valor encontrado para el cobre fue de $40,013 \mu \mathrm{g} / \mathrm{g}$, parece tener un origen más residual que no residual, lo que indica que el cobre en este caso forma parte de la estructura reticular de los componentes minerales de los sedimentos. Los no residuales son aquellos metales que se han ido incorporando a los sedimentos de soluciones acuosas, por medio de diferentes procesos, tales como adsorción, compleja-ción química e intercambio iónico (CHESTER \& VOUTSINOU, 1981, citados por Martinez G. 2002).

El zinc encontrado fue de $12,38 \mu \mathrm{g} / \mathrm{g}$, Además se señala que el zinc presente en sedimentos costeros tiene un origen litogénico al igual que el hierro y el manganeso (Martínez G. 2002). La Ley general de aguas D.L. 17752, y D.S. N ${ }^{\circ}$ 261-69-AP, en su Ítem V, Aguas de zonas de pesca de mariscos y bivalvos, establece límites de $20 \mu \mathrm{g} / \mathrm{l}$, para el zinc.

Valores de fierro encontrados en los sedimentos fueron de $1,8 \mu \mathrm{g} / \mathrm{g}$, son valores normales en esta zona. La existencia de valores altos de metales pesados se debe a que existe afinidad de estos para acumularse y ser transportados por los sólidos suspendidos, y una vez que entran en contacto con el medio marino son liberados debido al proceso de floculación que se da por la influencia del agua de mar sobre la del rio (Acosta V. et al. 2002). Debido a que en la zona no hay descargas considerables de rios, se tendría bajos niveles de Fe en lazona. 


\section{CONCLUSIONES}

1. En la zona de Punta Mesa para el agua de mar, se han obtenido niveles bastante bajos de metales pesados, siendo el más elevado el de cobre con 12,34 ug/L. Los promedios de resultados obtenidos en agua de mar en la Zona de Punta Mesa al sur del foco contaminado son: cadmio $0,53 \mathrm{ug} / \mathrm{L}$, plomo $0,72 \mathrm{ug} / \mathrm{L}$, cobre $10,66 \mathrm{ug} / \mathrm{L}$ y hierro $0,36 \mathrm{ug} / \mathrm{L}$ son relativamente bajos

2. En la zona de Punta mesa para sedimentos marinos se obtuvo el nivel más alto en manganeso con $164,50 \mathrm{ug} / \mathrm{g}$, y en segundo lugar el cobre con $46,84 \mathrm{ug} / \mathrm{g}$. Los promedios de resultados obtenidos en sedimentos marinos en la zona sur al foco contaminado son cadmio $0,16 \mathrm{ug} / \mathrm{g}$, plomo $1,44 \mathrm{ug} / \mathrm{g}$, manganeso $127,54 \mathrm{ug} / \mathrm{g}$, cobre 40,013 ug/g,zinc 12,38 ug/g y fierro $1,8 \mathrm{ug} / \mathrm{g}$ son valores normales en esta zona.

3. En la zona de muestreo 3, Santa Rosa, se ha obtenido el valor más alto en cobre con $15,96 \mathrm{ug} / \mathrm{g}$, mucho más alto que en la zona sur, otro nivel alto para un análisis de fierro con 15,73 ug/L., Los demás valores encontrados están al nivel de trazas. Los promedios de resultados obtenidos en agua de mar en la zona de Santa Rosa son: cadmio 0,53 ug/L, plomo $0,71 \mathrm{ug} / \mathrm{L}$, cobre $13,42 \mathrm{ug} / \mathrm{L}$ y hierro $1,605 \mathrm{ug} / \mathrm{L}$, son relativamente similares a los encontrados en la Zona sur.

4. Los niveles de metales pesados en la Zona de Santa Rosa en sedimentos marinos, según los resultados obtenidos, puede indicarse que el nivel mas alto encontrado es el cobre con 216,94 ug/g, seguido del manganeso con $85,84 \mathrm{ug} / \mathrm{g}$, y el zinc con $15,06 \mathrm{ug} / \mathrm{g}$, todos estos muy superiores a los encontrados en la Zona sur (libre de contaminación).

5. Los promedios de resultados obtenidos en sedimentos marinos en la Zona Norte al foco contaminado son cadmio $0,328 \mathrm{ug} / \mathrm{g}$, plomo 1,2 $\mathrm{ug} / \mathrm{g}$, manganeso $71,54 \mathrm{ug} / \mathrm{g}$, cobre 157,568 $\mathrm{ug} / \mathrm{g}$, zinc $9,94 \mathrm{ug} / \mathrm{g}$ y fierro $1,4 \mathrm{ug} / \mathrm{g}$. El valor que se encuentra por encima del valor encontrado en la Zona sur es el Cobre con una diferencia considerable.

\section{RECOMENDACIONES}

Continuar con los estudios, de metales pesados en diversos organismos de la fauna y flora marina de la zona de la Bahía de Ite y de todas las costas de Tacna.

Que la empresa responsable de haber vertido los relaves en la zona de playa ingresa entre (Bahía de Ite), ejecute un verdadero programa de remediación en la zona.
Que la empresa responsable permita el libre acceso a la zona contaminada para realizar estudios del nivel de contaminación real en que se encuentra la zona, ya que en la actualidad el acceso es restringido y se permite la toma de muestra en la zona del litoral.

\section{BIBLOGRAFÍA}

Arias de Diaz, Alicia; y Garcia, José. 2001. Concentración de los metales pesados Cu y Pb y su relación con la actividad enzimática glucógeno fosforilasa y gilucógeno sintetasa en el mejillón (Perna viridis), Zootecnia Trop., 19(2): 115-129.

Bartolini, R. 1988. La fertilidad de los suelos. Ediciones Mundi Prensa, Madrid. 140 pp.

Balbín Diaz, Doris, 1995. Agua, minería y contaminación: El caso Southern Perú. Editorial Labor, Ilo, Perú.

Cabrera C.,C.; Maldonado, D.M. Romero, Ch. L. 2004. Impacto ambiental en sedimentos marinos superficiales por residuos líquidos industriales y urbanos. Instituto de Investigaciones FIGMMG, Vol 7, 15-22, UNMSM, Lima.

Castro de Esparza, Maria Luisa. 2004. Arsénico en el agua de bebida de América Latina y su efecto en la salud pública. CEPIS/OPS.

FAO/WHO. 1996. Draft Principles and Guidelines for the Conduct of Microbiological Risk Assessment. CAC/GL-30 (1999). Food and Agriculture Organization of the United Nations, Rome, Italy.

García Cambero,J.P. Estado actual de la contaminación por metales pesados y pesticidas organoclorados en el parque natural de Monfrague. Memoria para optar el grado de Doctor, Facultad de Veterinaria, Universidad de Extremadura, España.

Hernández Moreno, D; Garcia F.M.A. Melgar Riol, M.; Pérez Lopez, M. (2004) "Estudio comparativo sobre el contenido de metales pesados en berbechos Cerastoderma edule $L$. en conserva y en fresco comercializados en España". Revista de Tecnologia e Higiene de Alimentos, Area de Toxicologia, Facultad de Veterinaria USC. Madrid.

Juárez Soto Henry Saúl, (2006) Contaminación del rio Rimac por metales pesados y efecto en la agricultura en el cono este de Lima Metropolitana. Reporte final de Investigación UNA, La Molina, Lima.

Lafuente G. Maria Asunción (2001), "Riesgos toxicológicos por exposición a metales 
pesados: Posibles marcadores de exposición", Rev. Toxicol.18, XIV Cong. Esp. de Toxicología

Marcos Pérez-López, Francisco Cid, Ana Lourdes Oropesa Luís Eusebio Hidalgo, Ana López Becerro, y Francisco Soler. Heavy metal and arsenic content in seabirds affected by the Prestige oil on the Gacilcian coast (NW Spain) (2005). Science of the Total Environment.

Moraga R, Merino C. y Mondaca, M.A. (2003) "Resistencia a metales pesados en bacterias aisladas de labahía de lquique". Investigaciones Marinas, Vol. 31(1) 2003.

Ministerio de Pesqueria, Dirección Regional Tacna, Corporación Departamental de Desarrollo (1984). Estudio contaminación recursos hídricos e hidrobiológicos, Tacna. Volumen II, Tacna, Perú.

Miranda, N. (2000) Tecnología de aguas: Control de calidad de aguas, Editorial Publicaciones, UNA, Puno, Perú. $128 \mathrm{pp}$.

OMS, (1984). Guía de calidad del agua para consumo humano, Organización Mundial de la Salud, Lima.

Paez-Ozuna, F., Botello, V.A. y Villanueva, F.S. (1986) "Heavy metals in Cotzacoalcos and ostión lagoon", México. Marine Pollution Bulletin. Vol.17, 11, Pages 516-519.

Pérez-López, M.; Alonso J.; Novoa-Viliñas M. C. y Melgar, M.J. (2003) Assessment of Heavy Metal Contamination of Seawater and Marine Limpet, Patella Vulgata L., from Northwest Spain. Journal of Environmental Science and Health, A38, pp.2845-2856.

Pérez-López, M.; Bellon, E.; Alonso, J.; y Melgar, M.J.; "Niveles de metales pesados y repercución toxicológica en conservas de mejillón (Mytilus sp)". Cien, y Tecnol. Aliment., Vol.4, 1, 22-27.
PNUMA: Diagnóstico regional sobre las actividades y fuentes terrestres de contaminación que afectan los ambientes marino, costero y dulceacuicola asociados en el Pacifico Sudeste. PNUMA / PAM Oficina de Coordinación y CPPS (1999). 73 pp.

Ramírez, AV, y Castillo EC. Arsenicismo ocupacional en metalurgia extractiva primaria. Salud, Trabajo y Medio Ambiente. Montevideo: 1998. p. 11.

Ramirez, Augusto V. (2006). Biomarcadores en monitoreo de exposición a metales pesados en metalurgia, An. Facc. de Medicina, Vol. $67 \mathrm{~N} 1$. The National Academy of Sciences, 1999. Arsenic in drinking water. National Academy Press, Washington, 1999. (Citado 2007 Enero. 05).

Http://books.nap.edu/books/0309063337/html 11.html

Vásquez A.V. (2000). Manejo de cuencas altoandino, Tomo 2, Universidad Agraria La Molina, Lima. 516 pp.

Villanueva F. Suzana; y Botello, Alfonso V (1992). "Metales pesados en la zona costera del golfo de México y Caribe mexicano: una revisión", Rev. Int. Contam. Ambient. 8 (I), 4761.1992

WHO. 1992a. Cadmium. Environmental Health Criteria 134. World Health Organization http://www.inchem.org/documents/ehc/ehc/eh c134.htm .

WHO. 1995. Inorganic lead. Environmental health criteria 165 . World Health Organization http://www.inchem.org/documents/ehc/ehc/eh c165.htm.

WHO - WORLD HEALTH ORGANIZATION. Mercury Environmental Aspects. Geneva: WHO, 1989. Environmental Health Criteria 86; $115 p$. 